\title{
Mapping the mechanisms of transcranial alternating current stimulation: a pathway from network effects to cognition
}

\author{
Department of Experimental Psychology, University of Oxford, Oxford, UK \\ ${ }^{*}$ Correspondence: ruairidh.battleday@sjc.ox.ac.uk \\ Edited by: \\ Matteo Feurra, National Research University Higher School of Economics, Russia \\ Reviewed by: \\ Matteo Feurra, National Research University Higher School of Economics, Russia \\ Cornelia Pirulli, IRCCS Centro San Giovanni di Dio Fatebenefratelli, Italy
}

Ruairidh M. Battleday*, Timothy Muller, Michael S. Clayton and Roi Cohen Kadosh

Keywords: neuroenhancement, transcranial electrical stimulation, transcranial alternating current stimulation, non-invasive brain stimulation, cognitive enhancement, oscillations, oscillatory activity

\section{INTRODUCTION}

In recent decades, our appreciation of the complexity of the brain has deepened immensely, as has our understanding of how it performs key functions. In the face of such complexity, and given the rising cost of neuropsychiatric illness (1), an intriguing question is whether we can promote further understanding, and in some cases enhancement, of the typical and atypical brain by targeted modulation of its activity. Notably, transcranial alternating current stimulation (tACS) which involves transcranial application of weak sinusoidal electrical currents (2) seems ideally suited to address this question, as it has been demonstrated to modulate endogenous oscillatory electrical activity (3), enhance cognitive functions (4-7), and provide support in neurological disease $(8,9)$. However, a complete mechanistic pathway between the neuronal and cognitive effects of tACS remains in need of explication, precluding both significant theoretical contribution by tACS studies, and the development of more adaptive neuroenhancement regimes. Therefore, in this Opinion article, we briefly review the role of oscillatory neuronal activity in cognition, before outlining one potential pathway by which the interaction between tACS and endogenous oscillations at a network level may be reconciled with its effects on broader cognitive functions.

\section{THE ROLES OF CORTICAL}

\section{OSCILLATIONS IN COGNITION}

Synchronous oscillations in the firing rates of large populations of neurons - recorded as fluctuations in electrical field potentials - represent a highly organized form of brain activity (see Figure 1). Specific oscillatory frequencies emerge in a task-, area-, and state-dependent manner, and are thought to reflect structural and functional features of the active neuronal networks mediating local and long-range cortical functions, and their cognitive manifestations [see in Ref. (10)]. For example, alpha oscillations (8$12 \mathrm{~Hz}$ ) over occipito-parietal cortices have been suggested to facilitate the inhibition of task-irrelevant visual processing (11), while increased oscillatory synchronization between frontal and parietal cortices in multiple frequency bands is thought critical to the orientation of attention (12) and the maintenance of working memory (13). Importantly, reliable alterations in oscillatory activity also feature in the pathophysiology of several neurological and psychiatric conditions (14-17).

\section{THE INTERACTION BETWEEN TACS AND ENDOGENOUS OSCILLATIONS}

Although the currents applied in tACS reliably generate fluctuations in individual neuronal firing rates (18), their interaction with larger ensembles in humans remains less clear. Nevertheless, two mechanistic insights have been derived from animal, computational, and human behavioral studies [see in Ref. $(18,19)]$ : (1) that in certain situations tACS may lock endogenous oscillatory activity to its frequency and phase ("entrainment"; see Figure 1); and, (2) that it may amplify a network's activity by stimulation at its "natural" frequency ("resonance"). However, the extent to which the behavioral and electrophysiological effects of tACS depend on these interactions remains unclear. As clarifying this issue is critical for full mechanistic understanding of tACS, and due to the recent publication of several studies that inform this debate, we review these theories below, before attempting to identify sufficient commonality between them to allow a more mechanistic consideration of the effects of tACS on cognition.

Entrainment refers to the synchronization of the frequency and phase of neuronal activity to external stimuli, such as rhythmic visual stimulation (20), or activity in other brain modules. In support of the theory that tACS entrains endogenous activity, weak alternating currents have been shown to shift the frequency and phase of neuronal firing in vivo, in vitro, and in computational models (18, 21-25). According to these computational accounts, entrainment increases the number of individual neurons firing at the peak of a particular oscillation - and therefore its power - in a manner that could conceivably affect behavior $(18,23)$. This theory has been corroborated by two recent human studies, the first of which reported increases in endogenous parieto-occipital alpha power during alpha-tACS to the same regions, noting that specific phases of tACS correlated with improved accuracy on a visual oddball task (3). The second reported that induction of lucid dreaming occurred with increases in endogenous fronto-temporal activity at 25 and $40 \mathrm{~Hz}$ during 25 and $40 \mathrm{~Hz}$ stimulation, 


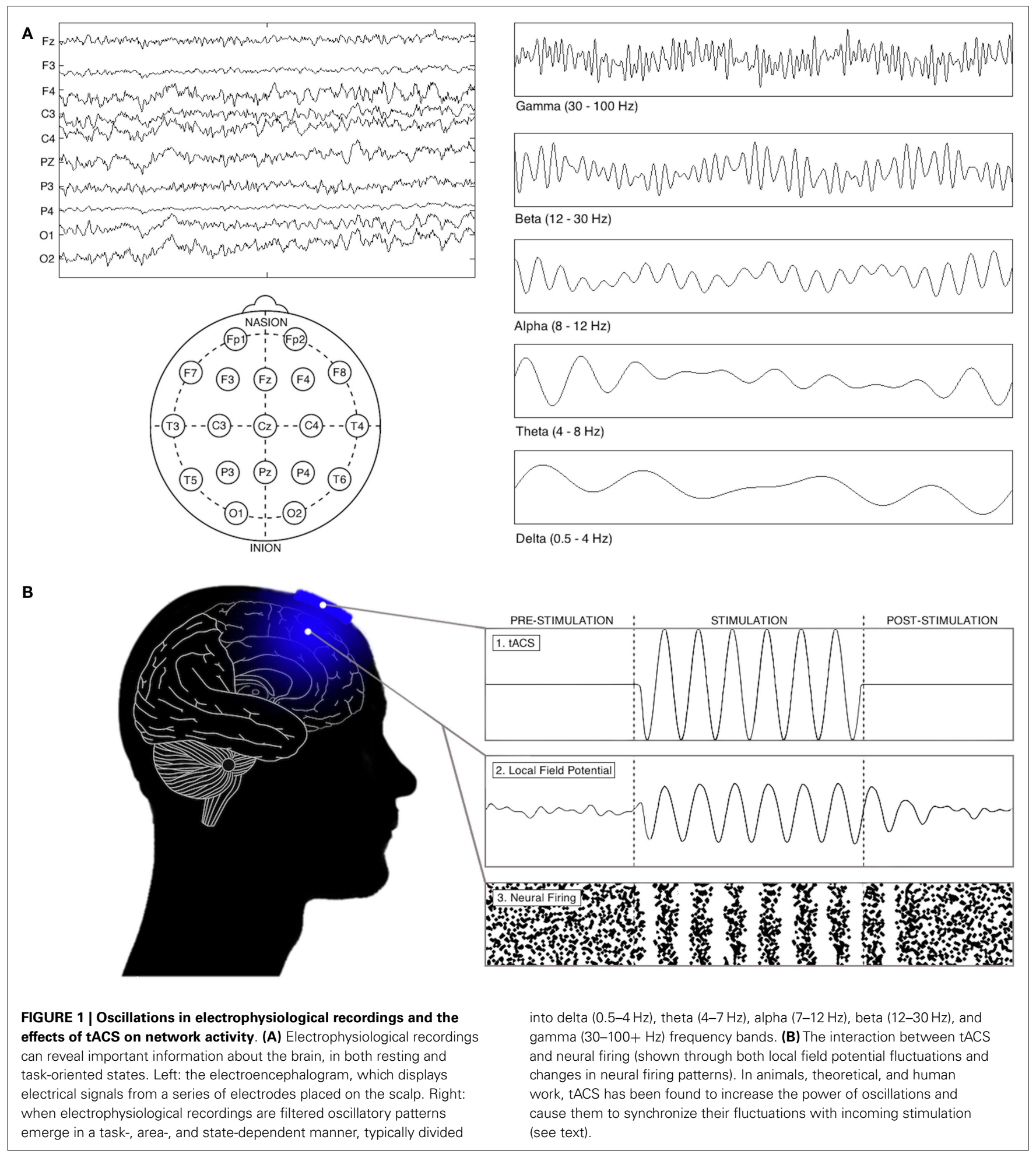

respectively (26). Indirect illustrations of the benefits of artificially entraining two endogenous oscillating systems have also been provided: Polania and colleagues reported decreased reaction times on a working memory task during $6 \mathrm{~Hz}$ tACS to left prefrontal and parietal cortices with $0^{\circ}$ phase alignment ("phasic"), and increased reaction times during tACS with $180^{\circ}$ phase alignment ("anti-phasic") (4). Similarly, Strüber and colleagues reported that anti-phasic $40 \mathrm{~Hz}$ tACS delivered to both parieto-occipital cortices caused perceptual alteration, whereas phasic tACS did not (27).

Two things are striking about the majority of tACS studies, including those mentioned above: (1) behavioral and 
electrophysiological amplification is typically only elicited at a single stimulation frequency; and, (2) this tends to be the frequency that dominates electrophysiological recordings of unstimulated subjects completing similar tasks, during similar behavioral states. For example, Feurra and colleagues showed that beta tACS of the motor cortex - which oscillates at beta frequencies during rest - but not stimulation at other frequencies, or to other areas, directly influenced motor cortex electrophysiology (28). Further, groups have shown that in the somatosensory (29) and visual (30) systems, tACS only elicits behavioral effects at frequencies similar to those recorded from the cortex during analogous sensory stimulation. Although it was initially argued that effects reported in the visual paradigm - namely, that phosphene perception was induced by alpha-tACS to the visual cortex in the dark, and beta $(12-30 \mathrm{~Hz})$ in the light, the dominant oscillations recorded in this area in dark and light environments, respectivelycould instead result from retinal activation (31-33), Kanai and colleagues subsequently showed that beta tACS of occipital but not frontal cortex directly interacts with underlying neuronal activity (34), casting doubt on the contribution of any volume conduction effect. Thus, it would seem that - rather than shifting the frequency and phase of endogenous oscillations - the causal interaction between tACS and neuronal networks depends on matching stimulation frequency with endogenous activity. According to the phenomenon of resonance, if task-activated oscillatory brain networks were stimulated at their resting frequency this could lead to augmentation of their activity (35). In support of this theory, Rosanova and colleagues showed that cortico-thalamic modules resonate at distinct frequencies if perturbed using transcranial magnetic stimulation (namely, alpha-band in occipital cortex, beta-band in parietal, and fast-beta-/gamma-band in frontal) (36). Further, Schmidt and colleagues showed that applying low intensity alternating electrical fields to an optogenetically induced "endogenous" $1 \mathrm{~Hz}$ firing rhythm in mouse neocortical slices preferentially increased oscillatory activity at $1 \mathrm{~Hz}$ and its first harmonic $(2 \mathrm{~Hz})$, collaborating model-predicted tACS-induced resonance phenomena $(22,37)$. Interestingly, stimulation using higher currents abolished this selectivity, implying that the ability of stimulation to drive network activity may be less constrained by the natural frequency of an area at higher intensities (38).

Although the entrainment and resonance accounts appear discrete, a common theme can be identified between them: the most important network effect of tACS is the wider recruitment of a - presumably previously partially enlisted - population of neurons into task-relevant rhythmically firing networks, occurring as a corollary of tightened synchrony or resonance properties. In this context, phase alignment between stimulation and endogenous oscillations is still important, as stipulated by theoretical accounts of entrainment (23) and resonance (39), and demonstrated by the human behavioral studies discussed above $(3,4,26,27)$. Thus, we propose that - at the low intensities applied using tACS - matching stimulation and endogenous frequencies are a necessary condition to enable the causal amplification of task-specific network activity, likely through an interaction that involves entrainment and resonance. This matching may proceed gradually, via entrainment, or may be imparted based on concurrent electrophysiology.

\section{MOVING FROM NETWORK EFFECTS TO COGNITIVE FUNCTIONS}

Further investigation of the network effects of tACS is clearly required; however, the question of how recruitment of a larger population of neurons oscillating at a particular frequency might affect a nebulous function like cognition may still be approached. To do so succinctly, the following premises must first be accepted: that particular cognitive functions are achieved by the activity of distributed brain networks; that particular cortical regions perform sub-computations of these functions; and that oscillations within brain tissue selectively enhance transfer of information through this network [see in Ref. $(10,40)$ ].

Since tACS targets a relatively localized cortical region, causing more widespread enlistment into a task- and state-activated rhythm should have direct consequences on information processing by that area. Neurons are more likely to fire in response to each other if they are synchronized, as input is more likely to be transmitted at periods of mutual depolarization (41). Thus, it is possible that tACS-induced increases in local coherent activity enhance information transfer and processing within the subset of oscillating networks that subserve task functions, thereby altering their contribution to cognitive processes. For example, given the hypothesized role of alpha rhythms in irrelevant visual input suppression (11), increasing the power of alpha oscillations could enhance the suppression of task-irrelevant visual inputs, and improve overall performance on visual attention tasks. This could conceivably have occurred in Helfrich and colleagues' study, where alpha-tACS increased alpha power over parieto-occipital cortices and improved performance in a visual task (3). Equally, if the inhibitory-excitatory balance or physical orientation of enlisted neurons were to differ markedly from the already active neuronal population, they could alter computations performed by that network; indeed, the sensitivity of neurons to applied electrical fields appears dependent on whether they are inhibitory or excitatory (42), and their orientation and geometry $(43,44)$. In this context, Brignani and colleagues' report of deterioration of visual performance following alpha or theta tACS to visual cortex regardless which hemisphere was stimulated warrants further investigation, as it questions the degree to which the effects of tACS can be considered local or frequency-specific (45).

As it is now thought that distributed cortico-thalamic networks mediate the computations underlying broad cognitive functions (46), it follows that tACS could also engender cognitive enhancement through modification of an area's function within such a network. Such an interaction could explain the reduction in the time taken to solve Raven's Matrices - a test of fluid intelligence, a function purportedly mediated by a multi-region network (47) - following gamma tACS of the left frontal lobe (in this case $40 \mathrm{~Hz}$ ) (6). According to Fries's communicationthrough-coherence hypothesis, information is passed more efficiently between network components if they are "coupled" to each other through synchronous depolarizations. Coupling could be established by increased synchronization of a region's output onto a recipient area, which would increase the impact of its output due to 
the time dependence of post-synaptic summation, and if sufficient lead to entrainment (40). It follows that by increasing synchrony in one area, tACS could affect its coupling with other network components, and thereby alter the cognitive function that emerges from the activity of that network. Notably, this theory predicts the results of Polanía and colleagues' study, in which two areas were artificially coupled at phasic and anti-phasic alignments (4). Finally, amplification of oscillatory power could alter synaptic weighting within the components of a network, potentially in turn augmenting and sustaining direct amplification of oscillatory activity (48).

\section{DISCUSSION}

In summary, by virtue of entrainment and resonance, tACS may modulate cognitive functions by enlisting a wider population of neurons into a local oscillating network, and in turn alter both the internal computations performed by an area and activity within a wider cerebral network. A critical question remains whether tACS can only be used to modulate one network per area per task. If this is the case, future improvements of tACS will depend on cataloging the spatio-temporal evolution of oscillatory patterns during task completion, as well as improving technology for applying tACS in single and multiple areas (49). If, instead, a number of networks may be selectively enlisted by stimulation at their distinct frequencies, the potential for probing and enhancing cerebral network function is vast. In either case, striving for a full mechanistic account of tACS remains vital in enabling tACS to be applied with maximal efficacy, in healthy and ill populations alike.

\section{REFERENCES}

1. Insel TR. Assessing the economic costs of serious mental illness. Am J Psychiatry (2008) 165:663-5. doi:10.1176/appi.ajp.2008.08030366

2. Antal A, Boros K, Poreisz C, Chaieb L, Terney D, Paulus W. Comparatively weak after-effects of transcranial alternating current stimulation (tACS) on cortical excitability in humans. Brain Stimul (2008) 1:97-105. doi:10.1016/j.brs.2007.10. 001

3. Helfrich RF, Schneider TR, Rach S, TrautmannLengsfeld SA, Engel AK, Herrmann CS. Entrainment of brain oscillations by transcranial alternating current stimulation. Curr Biol (2014) 24:333-9. doi:10.1016/j.cub.2013.12.041
4. Polanía R, Nitsche MA, Korman C, Batsikadze G, Paulus W. The importance of timing in segregated theta phase-coupling for cognitive performance. Curr Biol (2012) 22:1314-8. doi:10.1016/j. cub.2012.05.021

5. Pahor A, Jaušovec N. The effects of theta transcranial alternating current stimulation (tACS) on fluid intelligence. Int J Psychophysiol (2014) 93:322-31. doi:10.1016/j.ijpsycho.2014.06.015

6. Santarnecchi E, Polizzotto NR, Godone M, Giovannelli F, Feurra M, Matzen L, et al. Frequencydependent enhancement of fluid intelligence induced by transcranial oscillatory potentials. Curr Biol (2013) 23:1449-53. doi:10.1016/j.cub.2013. 06.022

7. Kar K, Krekelberg B. Transcranial alternating current stimulation attenuates visual motion adaptation. J Neurosci (2014) 34:7334-40. doi:10.1523/ JNEUROSCI.5248-13.2014

8. Brittain J-S, Probert-Smith P, Aziz TZ, Brown P. Tremor suppression by rhythmic transcranial current stimulation. Curr Biol (2013) 23:436-40. doi:10.1016/j.cub.2013.01.068

9. Fedorov A, Chibisova Y, Szymaszek A, Alexandrov M, Gall C, Sabel BA. Non-invasive alternating current stimulation induces recovery from stroke. Restor Neurol Neurosci (2010) 28:825-33. doi:10.3233/RNN-2010-0580

10. Wang X-J. Neurophysiological and computational principles of cortical rhythms in cognition. Physiol Rev (2010) 90:1195-268. doi:10.1152/physrev. 00035.2008

11. Jensen OMA. Shaping functional architecture by oscillatory alpha activity: gating by inhibition. Front Hum Neurosci (2010) 4:186. doi:10.3389/ fnhum.2010.00186

12. Daitch AL, Sharma M, Roland JL, Astafiev SV, Bundy DT, Gaona CM, et al. Frequency-specific mechanism links human brain networks for spatial attention. Proc Natl Acad Sci U S A (2013) 110(48):19585-90. doi:10.1073/pnas.1307947110

13. Salazar RF, Dotson NM, Bressler SL, Gray CM Content-specific fronto-parietal synchronization during visual working memory. Science (2012) 338:1097-100. doi:10.1126/science. 1224000

14. Brown P, Oliviero A, Mazzone P, Insola A, Tonali P, Di Lazzaro V. Dopamine dependency of oscillations between subthalamic nucleus and pallidum in Parkinson's disease. J Neurosci (2001) 21:1033-8.

15. Worrell GA, Parish L, Cranstoun SD, Jonas R, Baltuch G, Litt B. High-frequency oscillations and seizure generation in neocortical epilepsy. Brain (2004) 127:1496-506. doi:10.1093/brain/awh149

16. Montez T, Poil S-S, Jones BF, Manshanden I, Verbunt JP, van Dijk BW, et al. Altered temporal correlations in parietal alpha and prefrontal theta oscillations in early-stage Alzheimer disease. Proc Natl Acad Sci U S A (2009) 106:1614-9. doi:10.1073/ pnas.0811699106

17. Uhlhaas PJ, Singer W. Abnormal neural oscillations and synchrony in schizophrenia. Nat Rev Neurosci (2010) 11:100-13. doi:10.1038/nrn2774

18. Reato D, Rahman A, Bikson M, Parra LC. Lowintensity electrical stimulation affects network dynamics by modulating population rate and spike timing. J Neurosci (2010) 30:15067-79. doi:10. 1523/JNEUROSCI.2059-10.2010
19. Reato D, Rahman A, Bikson M, Parra LC. Effects of weak transcranial alternating current stimulation on brain activity-a review of known mechanisms from animal studies. Front Hum Neurosci (2013) 7:687. doi:10.3389/fnhum.2013. 00687

20. De Graaf TA, Gross J, Paterson G, Rusch T, Sack AT, Thut G. Alpha-band rhythms in visual task performance: phase-locking by rhythmic sensory stimulation. PLoS One (2013) 8:e60035. doi:10.1371/ journal.pone.0060035

21. Fröhlich F, McCormick DA. Endogenous electric fields may guide neocortical network activity. $\mathrm{Neu}$ ron (2010) 67:129-43. doi:10.1016/j.neuron.2010. 06.005

22. Ali MM, Sellers KK, Fröhlich F. Transcranial alternating current stimulation modulates largescale cortical network activity by network resonance. J Neurosci (2013) 33:11262-75. doi:10. 1523/JNEUROSCI.5867-12.2013

23. Radman T, Su Y, An JH, Parra LC, Bikson M. Spike timing amplifies the effect of electric fields on neurons: implications for endogenous field effects. J Neurosci (2007) 27:3030-6. doi:10.1523/ JNEUROSCI.0095-07.2007

24. Ozen S, Sirota A, Belluscio MA, Anastassiou CA, Stark E, Koch C, et al. Transcranial electric stimulation entrains cortical neuronal populations in rats. J Neurosci (2010) 30:11476-85. doi:10.1523/ JNEUROSCI.5252-09.2010

25. Deans JK, Powell AD, Jefferys JGR. Sensitivity of coherent oscillations in rat hippocampus to AC electric fields. J Physiol (2007) 583:555-65. doi:10.1113/jphysiol.2007.137711

26. Voss U, Holzmann R, Hobson A, Paulus W, Koppehele-Gossel J, Klimke A, et al. Induction of self awareness in dreams through frontal low current stimulation of gamma activity. Nat Neurosci (2014) 17:810-2. doi:10.1038/nn.3719

27. Strüber D, Rach S, Trautmann-Lengsfeld SA, Engel AK, Herrmann CS. Antiphasic $40 \mathrm{~Hz}$ oscillatory current stimulation affects bistable motion perception. Brain Topogr (2014) 27:158-71. doi:10.1007/ s10548-013-0294-x

28. Feurra M, Bianco G, Santarnecchi E, Del Testa M, Rossi A, Rossi S. Frequency-dependent tuning of the human motor system induced by transcranial oscillatory potentials. J Neurosci (2011) 31:12165-70. doi:10.1523/JNEUROSCI.0978-11. 2011

29. Feurra M, Paulus W, Walsh V, Kanai R. Frequency specific modulation of human somatosensory cortex. Front Psychol (2011) 2:13. doi:10.3389/fpsyg. 2011.00013

30. Kanai R, Chaieb L, Antal A, Walsh V, Paulus W. Frequency-dependent electrical stimulation of the visual cortex. Curr Biol (2008) 18:1839-43. doi:10.1016/j.cub.2008.10.027

31. Schutter DJLG, Hortensius R. Retinal origin of phosphenes to transcranial alternating current stimulation. Clin Neurophysiol (2010) 121:1080-4. doi:10.1016/j.clinph.2009.10.038

32. Schwiedrzik CM. Retina or visual cortex? The site of phosphene induction by transcranial alternating current stimulation. Front Integr Neurosci (2009) 3:6. doi:10.3389/neuro.07.006.2009

33. Terhune DB, Song SM, Cohen Kadosh R. Transcranial alternating current stimulation reveals atypical 
$40 \mathrm{~Hz}$ phosphene thresholds in synaesthesia. Cortex (2015) 63:267-70. doi:10.1016/j.cortex.2014. 09.006

34. Kanai R, Paulus W, Walsh V. Transcranial alternating current stimulation (tACS) modulates cortical excitability as assessed by TMS-induced phosphene thresholds. Clin Neurophysiol (2010) 121:1551-4. doi:10.1016/j.clinph.2010.03.022

35. Buzsaki G. Rhythms of the Brain. New York: Oxford University Press (2006).

36. Rosanova M, Casali A, Bellina V, Resta F, Mariotti M, Massimini M. Natural frequencies of human corticothalamic circuits. J Neurosci (2009) 29:7679-85. doi:10.1523/JNEUROSCI.0445-09. 2009

37. Merlet I, Birot G, Salvador R, Molaee-Ardekani B, Mekonnen A, Soria-Frish A, et al. From oscillatory transcranial current stimulation to scalp EEG changes: a biophysical and physiological modeling study. PLoS One (2013) 8:e57330. doi:10.1371/ journal.pone.0057330

38. Schmidt SL, Iyengar AK, Foulser AA, Boyle MR, Fröhlich F. Endogenous cortical oscillations constrain neuromodulation by weak electric fields. Brain Stimul (2014). doi:10.1016/j.brs.2014. 07.033

39. Longtin A. Stochastic resonance in neuron models. J Stat Phys (1993) 70:309-27. doi:10.1007/ BF01053970

40. Fries P. A mechanism for cognitive dynamics: neuronal communication through neuronal coherence. Trends Cogn Sci (2005) 9:474-80. doi:10. 1016/j.tics.2005.08.011
41. Azouz R, Gray CM. Adaptive coincidence detection and dynamic gain control in visual cortical neurons in vivo. Neuron (2003) 37:513-23. doi:10.1016/S0896-6273(02)01186-8

42. Moliadze V, Atalay D, Antal A, Paulus W. Close to threshold transcranial electrical stimulation preferentially activates inhibitory networks before switching to excitation with higher intensities. Brain Stimul (2012) 5:505-11. doi:10.1016/j.brs. 2011.11.004

43. Bikson M, Inoue M, Akiyama H, Deans JK, Fox JE, Miyakawa $\mathrm{H}$, et al. Effects of uniform extracellular DC electric fields on excitability in rat hippocampal slices in vitro. J Physiol (2004) 557:175-90. doi:10.1113/jphysiol.2003.055772

44. Miranda PC, Mekonnen A, Salvador R, Ruffini G. The electric field in the cortex during transcranial current stimulation. Neuroimage (2013) 70:48-58. doi:10.1016/j.neuroimage.2012.12.034

45. Brignani D, Ruzzoli M, Mauri P, Miniussi C. Is transcranial alternating current stimulation effective in modulating brain oscillations? PLoS One (2013) 8:e56589. doi:10.1371/journal.pone.0056589

46. Sporns O, Chialvo DR, Kaiser M, Hilgetag CC. Organization, development and function of complex brain networks. Trends Cogn Sci (2004) 8:418-25. doi:10.1016/j.tics.2004.07.008

47. Jung RE, Haier RJ. The parieto-frontal integration theory (P-FIT) of intelligence: converging neuroimaging evidence. Behav Brain Sci (2007) 30:135-54. doi:10.1017/S0140525X07001185

48. Zaehle T, Rach S, Herrmann CS. Transcranial alternating current stimulation enhances individual alpha activity in human EEG. PLoS One (2010) 5:e13766. doi:10.1371/journal.pone. 0013766

49. Fröhlich F. Endogenous and exogenous electric fields as modifiers of brain activity: rational design of noninvasive brain stimulation with transcranial alternating current stimulation. Dialogues Clin Neurosci (2014) 16:93-102.

Conflict of Interest Statement: The authors declare that the research was conducted in the absence of any commercial or financial relationships that could be construed as a potential conflict of interest.

Received: 27 September 2014; accepted: 01 November 2014; published online: 20 November 2014.

Citation: Battleday RM, Muller T, Clayton MS and Cohen Kadosh $R$ (2014) Mapping the mechanisms of transcranial alternating current stimulation: a pathway from network effects to cognition. Front. Psychiatry 5:162. doi: 10.3389/fpsyt.2014.00162

This article was submitted to Neuropsychiatric Imaging and Stimulation, a section of the journal Frontiers in Psychiatry.

Copyright $(2014$ Battleday, Muller, Clayton and Cohen Kadosh. This is an open-access article distributed under the terms of the Creative Commons Attribution License (CC BY). The use, distribution or reproduction in other forums is permitted, provided the original author(s) or licensor are credited and that the original publication in this journal is cited, in accordance with accepted academic practice. No use, distribution or reproduction is permitted which does not comply with these terms. 